\title{
From Hexaphenylbenzene to $1,2,3,4,5,6-$ Hexacyclohexylcyclohexane
}

Marcel Dillenburger, ${ }^{\perp}$ Zijie Qiu, ${ }^{*}{ }^{\perp}$ Cheng-Wei Ju, Beate Müller, Svenja Morsbach, Dieter Schollmeyer, Akimitsu Narita,* and Klaus Müllen*

Cite This: J. Am. Chem. Soc. 2020, 142, 12916-12920

Read Online

\section{ACCESS |}

Wll Metrics \& More

Article Recommendations

Supporting Information

ABSTRACT: The hydrogenation of hexaphenylbenzene was studied, affording novel partially hydrogenated hexacyclohexylbenzene (HCB) as well as fully hydrogenated 1,2,3,4,5,6-hexacyclohexylcyclohexane (HCC) as an unprecedented "oligocyclohexyl" molecule. The reaction process was analyzed by mass spectrometry with atmospheric pressure chemical ionization and high-performance liquid chromatography. From a crude product mixture, two different crystals with flake- and block-shapes could be grown and analyzed by X-ray crystallography, revealing their structures as HCB and HCC. While a geared arrangement of cyclohexyl substitutes was found in HCB, two isomeric structures were identified in HCC crystal with chair and twist-boat conformations of the central cyclohexane.

$\mathrm{P}$ olyphenylenes (PPs) can give rise to a great variety of structures. $^{1-4}$ The linear case, poly-para-phenylene, is the prototype of a conjugated polymer, but PPs can also be extended into branched, hyperbranched, and even dendritic analogues." ${ }^{5,6}$ Branched PPs also serve as precursors of "graphenic" molecules such as nanographenes, graphene nanoribbons, and nanobelts through oxidative dehydrogenation. $^{7-13}$ On the contrary, related structures made from cyclohexylene and cyclohexyl as repeating units have attracted much less attention. The saturated counterpart of poly-paraphenylene, polycyclohexylene, can be synthesized through anionic polymerization of 1,3 -cyclohexadiene $e^{14-16}$ and subsequent hydrogenation, ${ }^{17}$ but it contains both 1,2 - and 1,4connections (Scheme 1A). As for oligomers, there are few studies on "oligocyclohexyls" higher than tercyclohexyl, ${ }^{18-21}$

Scheme 1. Synthesis of (A) Polycyclohexylene, (B) 1,2,3,4Tetracyclohexylcyclohexane, (C) Hexacyclohexylbenzene (HCB), and 1,2,3,4,5,6-Hexacyclohexylcyclohexane (HCC) by Hydrogenation
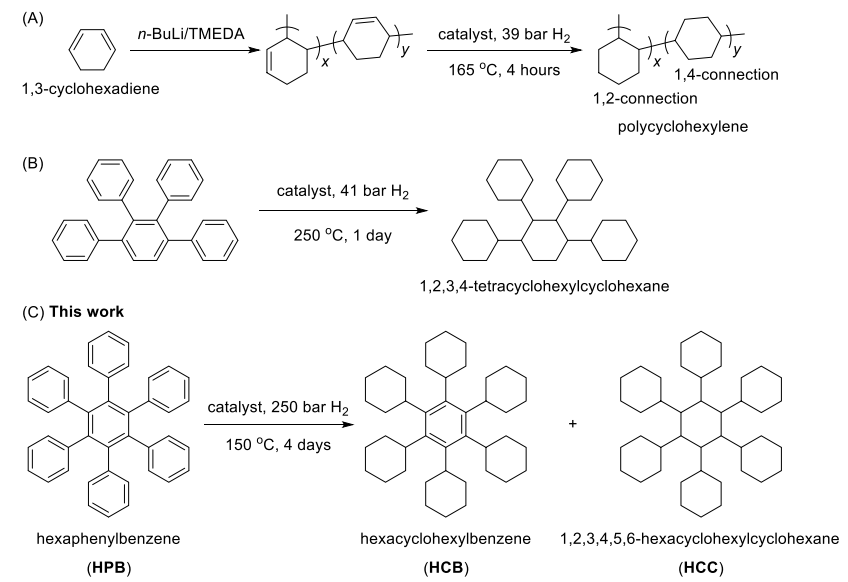

and 1,2,3,4-tetracyclohexylcyclohexane is the biggest reported example in the literature (Scheme $1 \mathrm{~B}){ }^{22}$

Hexaphenylbenzene (HPB) is the precursor of the "flattening" reaction toward hexa-peri-hexabenzocoronene $(\mathrm{HBC})^{23-26}$ and the core of important polyphenylene dendrimers. ${ }^{6}$ During our studies on the hydrogenation of $\mathrm{HBC}$ and larger nanographenes, ${ }^{27,28}$ our attention was directed to the hydrogenation of HPB, which could be the cornerstone for the future synthesis of fully saturated counterparts of polyphenylene dendrimers. ${ }^{29,30}$

In this communication, we report the synthesis of hexacyclohexylbenzene (HCB) and 1,2,3,4,5,6-hexacyclohexylcyclohexane (HCC) by hydrogenation of HPB (Scheme 1C). Mass spectrometry and high-performance liquid chromatography (HPLC) were used to investigate the reaction process. Although it is not possible to purify the hydrogenated products by conventional purification methods, two different crystals with flake- and block-shapes could be grown from a crude product mixture. The chemical structures of these two crystals were unambiguously confirmed as HCB and HCC by X-ray crystal analysis. While a "tongue and groove" arrangement of cyclohexyl substitutes was found in HCB, two isomeric structures, HCC-chair and HCC-TB, were identified in HCC crystal with different stereochemistry.

The hydrogenation of HPB was first carried out with commercially available $\mathrm{Pd} / \mathrm{C}$ as the catalyst. After sonication of $\mathrm{Pd} / \mathrm{C}$ together with $\mathrm{HPB}$ in cyclohexane for $1 \mathrm{~h}$, the reaction mixture was transferred into an autoclave and bubbled with a $\mathrm{H}_{2}$ flow for $10 \mathrm{~min}$. The autoclave was sealed with 210 bar of

Received: May 6, 2020

Published: July 15, 2020 
$\mathrm{H}_{2}$ at room temperature and then heated to $150{ }^{\circ} \mathrm{C}$ under stirring, which increased the pressure to 250 bar.

To monitor the reaction process, aliquots were taken from the reaction mixture after 1,2 , and 4 days for mass analysis. While matrix-assisted laser desorption/ionization (MALDI) mass spectrometry always provided results of the aromatized compounds due to the difficulties in ionizing the saturated nonpolar compounds and possible dehydrogenation under the conditions of the measurement, analysis with an atmospheric pressure chemical ionization (APCI-MS) ion source could provide initial information. As demonstrated by APCI-MS in Figure $1 \mathrm{~A}$, the starting HPB $\left(M_{\text {cal }}=534 \mathrm{Da}\right)$ was consumed
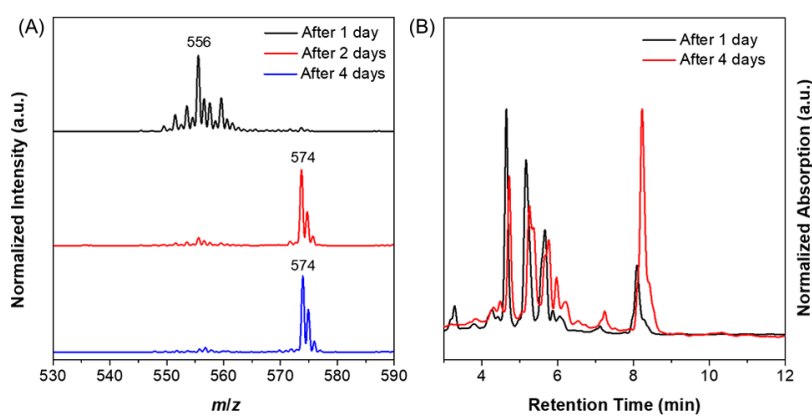

Figure 1. (A) Mass spectra of reaction mixtures after 1, 2, and 4 days using APCI ion source. (B) HPLC analysis of reaction mixtures after 1 and 4 days eluted using a gradient from tetrahydrofuran/acetonitrile (5\%/95\%) to tetrahydrofuran (100\%) over $20 \mathrm{~min}$ with a flow rate of $1 \mathrm{~mL} / \mathrm{min}$ on a Gemini $5 \mu \mathrm{m}$ C6-Phenyl column. The HPLC signal was taken from absorption at $220 \mathrm{~nm}$.

completely after 1 day, yielding a series of hydrogenated products. These intermediates had been further reacted after 2 days, where a major peak at $\mathrm{m} / z=574$ appeared, suggesting the formation of a completely hydrogenated product $\left(M_{\text {cal }}=\right.$ $576 \mathrm{Da})$. The difference of experimental and calculated molecular masses might be due to the loss of hydrogen atoms during the APCI-MS measurement. When extending the reaction time to 4 days, only a slight improvement was observed from the APCI-MS spectrum, and thus the hydrogenation reaction was terminated at the fourth day.

The crude products after 1 and 4 days were also analyzed by HPLC with a reversed phase column (Gemini $5 \mu$ m C6-Phenyl column), where compounds with less $\pi$-conjugation had longer retention time. After screening different solvent combinations, several peaks could be resolved by using a gradient eluent of tetrahydrofuran and acetonitrile (Figure 1B). Compared with the HPLC spectrum after 1 day, the peak with the longest retention time $(\sim 8 \mathrm{~min})$ was dramatically enhanced after 4 days. In combination with the APCI-MS analysis, the fully hydrogenated compound, HCC, was expected as the major product and assigned to that peak. Besides $\mathrm{Pd} / \mathrm{C}$, we have also tried two bimetallic catalysts prepared according to a literature method, ${ }^{31}$ namely $\mathrm{Pt}-\mathrm{Rh}$ nanoparticles and $\mathrm{Pd}-\mathrm{Rh}$ nanoparticles supported by carbon nanotubes ( $\mathrm{Pt}-\mathrm{Rh} / \mathrm{MWNTs}$ and $\mathrm{Pt}-\mathrm{Rh} / \mathrm{MWNTs}$ ). However, the peaks at a retention time of $\sim 8$ min were negligible in their HPLC analysis (Figure S1), thus indicating a lower efficiency than $\mathrm{Pd} / \mathrm{C}$ toward the fully hydrogenated product.

After removal of $\mathrm{Pd} / \mathrm{C}$ by filtration and a flash column of silica gel, the crude product was obtained as a colorless solid in $76 \mathrm{mg}$. Besides the peaks at the retention time of $\sim 8 \mathrm{~min}$ in the HPLC analysis, multiple peaks with shorter retention time still exist, suggesting byproducts with different degrees of hydrogenation. It is worth noting that the yield of fully hydrogenated HCC can not be estimated from the peak areas because of its low UV absorption. Because of the small difference between partially and fully hydrogenated compounds, purification upon conventional silica gel chromatography and large scale recrystallization were not possible. Fortunately, two different crystals with shapes of flakes and blocks could be grown by slow vapor diffusion of antisolvent methanol into the chloroform solution of the crude product (Figure S2). After careful mechanical separation of two kinds of tiny crystals, Xray crystallography results revealed the structures of these two crystals to be partially hydrogenated HCB and fully hydrogenated HCC after reaction.

The conformations of cyclohexane (chair, half-chair, boat, and twist-boat forms) are amply described as classics of organic stereochemistry, ${ }^{32-35}$ rendering the stereochemistry analysis of HCB and HCC particularly exciting. HCB forms monoclinic crystals with a molecular $C_{\mathrm{i}}$ symmetry (Figure 2A). All

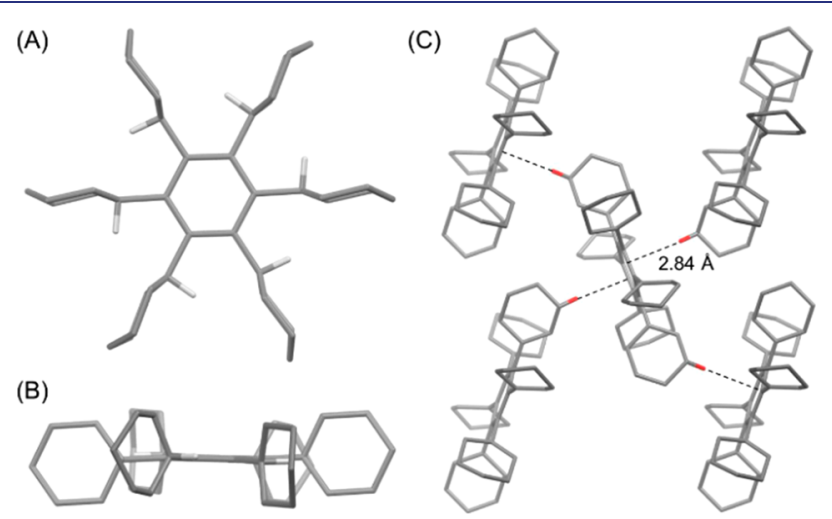

Figure 2. Single-crystal structure of HCB: (A) top view, (B) side view. Only the methine protons are shown for clarity. (C) Molecular packing of $\mathrm{HCB}$ with highlighting $\mathrm{CH} / \pi$ interactions.

cyclohexyl substituents adopt the well-known chair conformation and are almost perpendicularly attached to the central benzene ring. The latter remains planar without distortion as demonstrated in Figure 2B. In the molecular packing, $\mathrm{CH} / \pi$ interactions can be found between adjacent HCB molecules with a distance of $2.84 \AA$ (Figure $2 \mathrm{C}$ ). Notably, the methine hydrogens, adjacent to the benzene ring, of six cyclohexyl units are regularly oriented in the same direction of rotation, constituting a "tongue and groove" arrangement. Such static gearing was also observed in the early works of tetracyclohexylethene and hexaisopropylbenzene. ${ }^{36-38}$ Experimental determination of the cyclohexyl rotation, where isotopic labeling is required, ${ }^{37}$ was not achieved due to the lack of proper equipment. On the basis of a method described by Siegel et al., ${ }^{38}$ a moderate activation energy barrier $(26.8 \mathrm{kcal} /$ mol) was estimated by DFT calculations (Figures S4 and S5; Table S1), which was expected to allow rotation of cyclohexyl substituents upon heating.

By contrast, the cyclohexane conformations in the HCC crystal are rather complex. Two isomeric structures of HCC could be found (Figure 3A,B). The absolute configurations of the central cyclohexane in these two structures are identified as $1 R, 2 S, 3 S, 4 S, 5 S, 6 R$ and $1^{\prime} r, 2^{\prime} r, 3^{\prime} r, 4^{\prime} r, 5^{\prime} r, 6^{\prime} r$ according to the nomenclature rules, respectively (Figure $3 \mathrm{C}-\mathrm{D}$ ). The peripheral cyclohexyl units in both structures adopt chair 


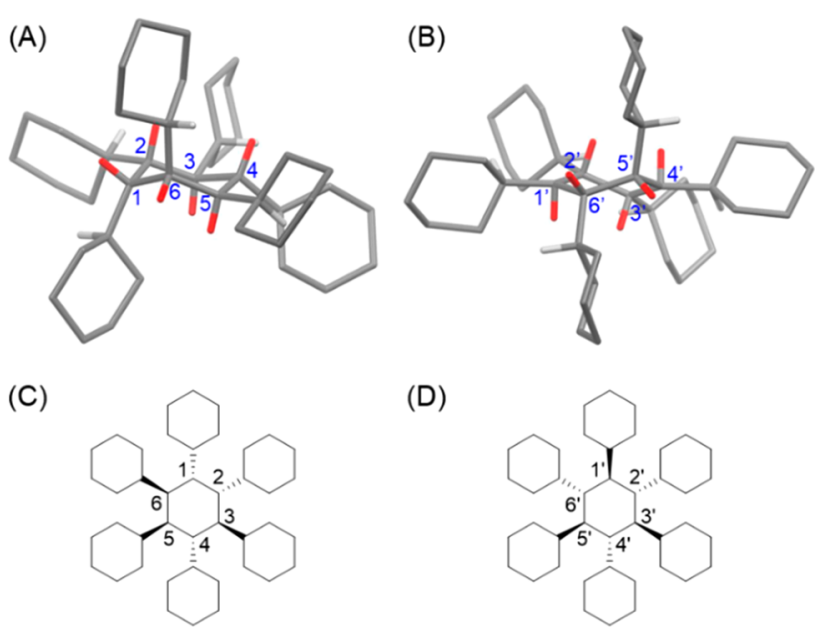

Figure 3. Single-crystal structures of HCC with (A) chair and (B) deformed conformations of the central cyclohexane. Only the methine protons are displayed for clarity. The methine protons in the central cyclohexane are highlighted in red for easy identification. Molecular configurations of (C) HCC-chair and (D) HCC-TB.

conformations. As shown in Figure 3A, the central cyclohexane in the first structure exhibits a chair form with average internal carbon bond angles of $112.4^{\circ}$, which is very close to that of the ideal chair of unsubstituted cyclohexane $\left(111.4^{\circ}\right)$. In the second isomer, the central cyclohexane is more deformed into a twisted-boat (TB) conformation. The sum of the six internal $\mathrm{C}-\mathrm{C}-\mathrm{C}-\mathrm{C}$ angles of torsion was calculated as $-4.5^{\circ}$, suggesting a flattened ring of the central cyclohexane (Figure $3 \mathrm{~B})$. Thus, these two isomeric structures are denoted as HCCchair and HCC-TB, respectively. The ratio between these two isomers is approximately 1:1 in the HCC crystal. As suggested by DFT calculations, the HCC-chair is more stable than HCCTB with a slightly lower Gibbs free energy of $3.38 \mathrm{~kJ} / \mathrm{mol}$ (Figure S5).

The formation of two different structures of HCC can be explained by the different orientations of methine protons in the central cyclohexane, which are highlighted in red in Figure $3 \mathrm{~A}$ and $\mathrm{B}$. The presence of two isomers indicates that there is no selectivity for the addition of hydrogen during reaction. Hydrogen can attack the benzene ring from both sides. Therefore, more isomeric structures of HCC are possible in the crude product, although they did not crystallize. The configurations of possible isomers are listed in Scheme S1. HCC molecules are loosely packed apparently without strong intermolecular interactions, with the shortest intermolecular $\mathrm{H}-\mathrm{H}$ distance of $2.28 \AA$, presumably due to its highly twisted conformation and the lack of $\pi$-conjugation or functional groups in its molecular structure (Figure S3).

To assign the peaks in the HPLC analysis of the crude product (Figures 1 and S1), the crystals of HCB and HCC were each dissolved in THF and subjected to HPLC analysis. By using the same HPLC condition as above, the retention time of HCB and HCC was determined to be $\sim 6$ and $8 \mathrm{~min}$, respectively (Figure 4A). Unfortunately, HCC-chair and HCC-TB could not be further differentiated. The absorption spectra of HCB and HCC were also measured by the UV-vis detector during HPLC analysis, showing absorption maxima in the UV region with peaks at 274 and $246 \mathrm{~nm}$, respectively (Figure 4B). The ${ }^{1} \mathrm{H}$ NMR spectra of $\mathrm{HCB}$ were measured after accumulating pure samples by careful recrystallization and
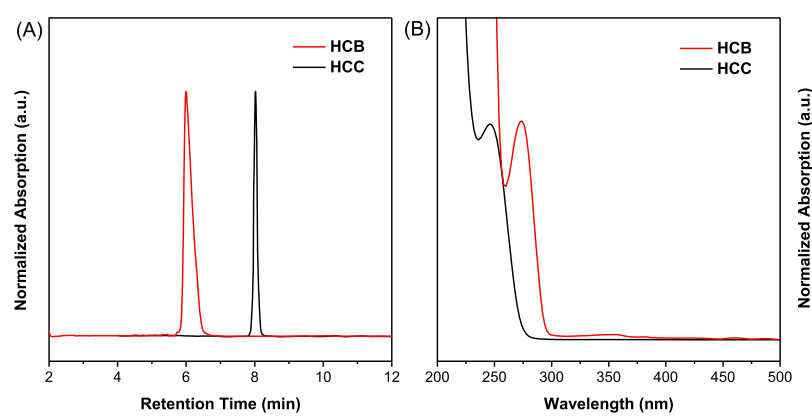

Figure 4. (A) HPLC analysis of HCB and HCC crystals eluted using a gradient from tetrahydrofuran/acetonitrile $(5 \% / 95 \%)$ to tetrahydrofuran $(100 \%)$ over $20 \mathrm{~min}$ with a flow rate of $1 \mathrm{~mL} / \mathrm{min}$ on a Gemini $5 \mu \mathrm{m}$ C6-Phenyl column. (B) Normalized absorption spectra of HCB and HCC measured from the UV-vis detector during HPLC analysis.

manual separation of the crystals with flake shape, while that of HCC was recorded for a sample accumulated by analytical HPLC. For HCB, the methine peak appears at $3.66 \mathrm{ppm}$, which, as expected, is shifted downfield relative to the signals of the other aliphatic protons (Figure S7). The integration of peaks at 3.66 and $1.79 \mathrm{ppm}$ is close to $1: 2$, which is supported by the simulated NMR spectrum by DFT calculation (Figure S8). However, only broad proton peaks were observed in the range of $0.8-1.8 \mathrm{ppm}$ for HCC (Figure S9), most probably due to the presence of various conformational isomers of cyclohexyl structures in solution, which is consistent with literature reports of polycyclohexylene and 1,2,3,4tetracyclohexylcyclohexane. ${ }^{17,22}$ Because of the tiny amounts of pure samples, the accurate yields and ${ }^{13} \mathrm{C}$ NMR spectra of HCB and HCC could not be obtained.

In conclusion, the hydrogenation of hexaphenylbenzene was successfully achieved, furnishing the partially hydrogenated hexacyclohexylbenzene (HCB) and the fully hydrogenated 1,2,3,4,5,6-hexacylohexylcyclohexane (HCC). Assisted by single-crystal analysis, their chemical structures could be determined. In the HCB crystal, six cyclohexyl substituents adopt chair conformations and are regularly oriented in a "tongue and groove" way. Two isomers, HCC-chair and HCCTB, could be found in the HCC crystal with distinct absolute configuration of the central cyclohexane, namely $1 R, 2 S, 3 S, 4 S, 5 S, 6 R$ and $1^{\prime} r, 2^{\prime} r, 3^{\prime} r, 4^{\prime} r, 5^{\prime} r, 6^{\prime} r$, respectively, which exhibited chair and twisted-boat structures. Although it is not possible in conventional purification methods, HCB and HCC could be separated into two peaks by HPLC with an analytical reversed phase column using a gradient elution but could not be fully resolved at a semipreparative scale after many attempts. The present work can be regarded as a model study for future syntheses of fully saturated counterparts of polyphenylene dendrimers through hydrogenation. Catalyst systems with higher hydrogenation efficiency are critical for future studies. The investigations using different heterogeneous and homogeneous catalysts, including state-of-the-art metal clusters and single-atom catalysts, are ongoing to achieve clean conversion toward fully hydrogenated compounds. Studies on the reversible dehydrogenation-hydrogenation process of these oligocyclohexyl compounds will hopefully allow their potential applications in hydrogen storage. ${ }^{39-41}$ 


\section{ASSOCIATED CONTENT}

\section{(3) Supporting Information}

The Supporting Information is available free of charge at https://pubs.acs.org/doi/10.1021/jacs.0c04956.

Experimental details, X-ray crystallography data, computational studies, ${ }^{1} \mathrm{H}$ NMR spectra (PDF)

Crystallographic data (CIF)

\section{AUTHOR INFORMATION}

\section{Corresponding Authors}

Zijie Qiu - Max Planck Institute for Polymer Research, 55128

Mainz, Germany; 이이이.org/0000-0003-0728-1178;

Email: qiu@mpip-mainz.mpg.de

Akimitsu Narita - Max Planck Institute for Polymer Research, 55128 Mainz, Germany; 이이이.org/0000-0002-3625522X; Email: narita@mpip-mainz.mpg.de

Klaus Müllen - Max Planck Institute for Polymer Research, 55128 Mainz, Germany; 이이.org/0000-0001-6630-

8786; Email: muellen@mpip-mainz.mpg.de

\section{Authors}

Marcel Dillenburger - Max Planck Institute for Polymer Research, 55128 Mainz, Germany

Cheng-Wei Ju - Max Planck Institute for Polymer Research, 55128 Mainz, Germany; College of Chemistry, Nankai University, Tianjin 300071, China

Beate Müller - Max Planck Institute for Polymer Research, 55128 Mainz, Germany

Svenja Morsbach - Max Planck Institute for Polymer Research, 55128 Mainz, Germany; (1) orcid.org/0000-0001-9662-8190

Dieter Schollmeyer - Department of Chemistry, Johannes Gutenberg-University, 55099 Mainz, Germany

Complete contact information is available at:

https://pubs.acs.org/10.1021/jacs.0c04956

\section{Author Contributions \\ ${ }^{\perp}$ M.D. and Z.Q. contributed equally.}

Notes

The authors declare no competing financial interest.

\section{ACKNOWLEDGMENTS}

This work was financially supported by the Max Planck Society and the Alexander von Humboldt Foundation.

\section{REFERENCES}

(1) Berresheim, A. J.; Müller, M.; Müllen, K. Polyphenylene Nanostructures. Chem. Rev. 1999, 99, 1747-1785.

(2) John, J. A.; Tour, J. M. Synthesis of Polyphenylene Derivatives by Thermolysis of Enediynes and Dialkynylaromatic Monomers. Tetrahedron 1997, 53, 15515-15534.

(3) Li, C.; Liu, M.; Pschirer, N. G.; Baumgarten, M.; Müllen, K. Polyphenylene-Based Materials for Organic Photovoltaics. Chem. Rev. 2010, 110, 6817-6855.

(4) Koga, Y.; Kaneda, T.; Saito, Y.; Murakami, K.; Itami, K. Synthesis of partially and fully fused polyaromatics by annulative chlorophenylene dimerization. Science 2018, 359, 435-439.

(5) Kim, Y. H.; Webster, O. W. Water-Soluble Hyperbranched Polyphenylene: "A Unimolecular Micelle"? J. Am. Chem. Soc. 1990, 112, 4592-4593.

(6) Hammer, B. A.; Müllen, K. Dimensional Evolution of Polyphenylenes: Expanding in All Directions. Chem. Rev. 2016, 116, 2103-2140.
(7) Narita, A.; Wang, X. Y.; Feng, X.; Müllen, K. New advances in nanographene chemistry. Chem. Soc. Rev. 2015, 44, 6616-6643.

(8) Kim, K. T.; Jung, J. W.; Jo, W. H. Synthesis of graphene nanoribbons with various widths and its application to thin-film transistor. Carbon 2013, 63, 202-209.

(9) Cheung, K. Y.; Gui, S.; Deng, C.; Liang, H.; Xia, Z.; Liu, Z.; Chi, L.; Miao, Q. Synthesis of Armchair and Chiral Carbon Nanobelts. Chem. 2019, 5, 838-847.

(10) Vo, T. H.; Shekhirev, M.; Kunkel, D. A.; Morton, M. D.; Berglund, E.; Kong, L.; Wilson, P. M.; Dowben, P. A.; Enders, A.; Sinitskii, A. Large-scale solution synthesis of narrow graphene nanoribbons. Nat. Commun. 2014, 5, 3189.

(11) Kim, Y. H. Hyperbranched Polymers 10 Years After. J. Polym. Sci., Part A: Polym. Chem. 1998, 36, 1685-1698.

(12) Sakaguchi, H.; Song, S.; Kojima, T.; Nakae, T. Homochiral polymerization-driven selective growth of graphene nanoribbons. Nat. Chem. 2017, 9, 57-63.

(13) Qiu, Z.; Narita, A.; Müllen, K. Carbon Nanostructures by Macromolecular Design - From Branched Polyphenylenes to Nanographenes and Graphene Nanoribbons. Faraday Discuss. 2020, DOI: $10.1039 /$ D0FD00023J.

(14) Natori, I.; Inoue, S. Anionic Polymerization of 1,3-Cyclohexadiene with Alkyllithium/Amine Systems. Characteristics of $n$ Butyllithium/ N,N, $\mathrm{N}^{\prime}, \mathrm{N}^{\prime}$-Tetramethylethylenediamine System for Living Anionic Polymerization. Macromolecules 1998, 31, 4687-4694.

(15) Natori, I. Synthesis of Polymers with an Alicyclic Structure in the Main Chain. Living Anionic Polymerization of 1,3-Cyclohexadiene with the n-Butyllithium/N,N, $\mathrm{N}^{\prime}, \mathrm{N}^{\prime}$-Tetramethylethylenediamine System. Macromolecules 1997, 30, 3696-3697.

(16) Natori, I.; Imaizumi, K.; Yamagishi, H.; Kazunori, M. Hydrocarbon Polymers Containing Six-Membered Rings in the Main Chain. Microstructure and Properties of Poly(1,3-cyclohexadiene). J. Polym. Sci., Part B: Polym. Phys. 1998, 36, 1657-1668.

(17) Natori, I. Hydrogenation of Poly(1,3-cyclohexadiene). Polym. J. 2003, 35, 622-627.

(18) Schmidt-Collérus, J. J.; Krimmel, J. A.; Stacy, R. D. Heptyl and Nonyl Derivatives of Bicyclohexyl and m-Tercyclohexyl. J. Org. Chem. 1961, 23, 716-718.

(19) Kalenchuk, A. N.; Koklin, A. E.; Bogdan, V. I.; Kustov, L. M. Hydrogenation of biphenyl and isomeric terphenyls over a Ptcontaining catalyst. Russ. Chem. Bull. 2017, 66, 1208-1212.

(20) Columbus, I.; Haj-Zaroubi, M.; Siegel, J. S.; Biali, S. E. Face Selectivity in the Hydrogenation of Calixarene Ethers. J. Org. Chem. 1998, 63, 9148-9149.

(21) Abu-Reziq, R.; Avnir, D.; Miloslavski, I.; Schumann, H.; Blum, J. Entrapment of metallic palladium and a rhodium(I) complex in a silica sol-gel matrix Formation of a highly active recyclable arene hydrogenation catalyst. J. Mol. Catal. A: Chem. 2002, 185, 179-185.

(22) Columbus, I.; Cohen, S.; Biali, S. E. Preparation and Molecular Structure Determination of a 1,2,3,4-Tetracyclohexylcyclohexane Existing in a Twist-Boat Conformation. J. Am. Chem. Soc. 1994, 116, 10306-10307.

(23) Gross, L.; Moresco, F.; Ruffieux, P.; Gourdon, A.; Joachim, C.; Rieder, K.-H. Tailoring molecular self-organization by chemical synthesis: Hexaphenylbenzene, hexa-peri-hexabenzocoronene, and derivatives on $\mathrm{Cu}$ (111). Phys. Rev. B: Condens. Matter Mater. Phys. 2005, 71, 165428.

(24) Suzuki, S.; Segawa, Y.; Itami, K.; Yamaguchi, J. Synthesis and characterization of hexaarylbenzenes with five or six different substituents enabled by programmed synthesis. Nat. Chem. 2015, 7, 227-233.

(25) Wadumethrige, S. H.; Rathore, R. A Facile Synthesis of Elusive Alkoxy-Substituted Hexa-peri-hexabenzocoronene. Org. Lett. 2008, $10,5139-5142$.

(26) Wang, Z.; Dotz, F.; Enkelmann, V.; Mullen, K. "Doubleconcave" $^{\prime \prime}$ graphene: permethoxylated hexa-peri-hexabenzocoronene and its cocrystals with hexafluorobenzene and fullerene. Angew. Chem., Int. Ed. 2005, 44, 1247-1250. 
(27) Yao, X.; Wang, X. Y.; Simpson, C.; Paterno, G. M.; Guizzardi, M.; Wagner, M.; Cerullo, G.; Scotognella, F.; Watson, M. D.; Narita, A.; Müllen, K. Regioselective Hydrogenation of a 60-Carbon Nanographene Molecule toward a Circumbiphenyl Core. J. Am. Chem. Soc. 2019, 141, 4230-4234.

(28) Watson, M. D.; Debije, M. G.; Warman, J. M.; Müllen, K. Peralkylated Coronenes via Regiospecific Hydrogenation of Hexaperi-hexabenzocoronenes. J. Am. Chem. Soc. 2004, 126, 766-771.

(29) Iyer, V. S.; Yoshimura, K.; Enkelmann, V.; Epsch, R.; Rabe, J. P.; Müllen, K. A Soluble C60 Graphite Segment. Angew. Chem., Int. Ed. 1998, 37, 2696-2699.

(30) Dotz, F.; Brand, J. D.; Ito, S.; Gherghel, L.; Müllen, K. Synthesis of Large Polycyclic Aromatic Hydrocarbons: Variation of Size and Periphery. J. Am. Chem. Soc. 2000, 122, 7707-7717.

(31) Pan, H.-B.; Wai, C. M. Facile sonochemical synthesis of carbon nanotube-supported bimetallic $\mathrm{Pt}-\mathrm{Rh}$ nanoparticles for room temperature hydrogenation of arenes. New J. Chem. 2011, 35, $1649-1660$.

(32) Columbus, I.; Biali, S. E. Stereochemistry of Polycyclohexyl Systems. Chirality 1998, 10, 159-168.

(33) Columbus, I.; Hoffman, R. E.; Biali, S. E. Stereochemistry and Conformational Anomalies of 1,2,3- and 1,2,3,4-Polycyclohexylcyclohexanes. J. Am. Chem. Soc. 1996, 118, 6890-6896.

(34) Eliel, E. L.; Wilen, S. H. Stereochemistry of Organic Compounds; Wiley: India, 2008.

(35) Columbus, I.; Biali, S. E. Cyclohexyl Rings in Spatial Proximity: Stereochemistry of Tricyclohexylmethane. J. Org. Chem. 1993, 58, 7029-7035.

(36) Columbus, I.; Biali, S. E. Gear Effects in Polycyclohexyl Systems: Tetracyclohexylethene. J. Org. Chem. 1994, 59, 3402-3407.

(37) Siegel, J.; Mislow, K. Experimental Evidence for a High Barrier to Internal Rotation in Hexaisopropylbenzene. J. Am. Chem. Soc. 1983, 105, 7763-7764.

(38) Siegel, J.; Gutierrez, A.; Schweizer, W. B.; Ermer, O.; Mislow, K. Static and Dynamic Stereochemistry of Hexaisopropylbenzene: A Gear-Meshed Hydrocarbon of Exceptional Rigidity. J. Am. Chem. Soc. 1986, 108, 1569-1575.

(39) Cacciola, G.; Giordano, N.; Restuccia, G. Cyclohexane as a liquid phase carrier in hydrogen storage and transport. Int. J. Hydrogen Energy 1984, 9, 411-419.

(40) Wang, Y.; Shah, N.; Huffman, G. P. Pure Hydrogen Production by Partial Dehydrogenation of Cyclohexane and Methylcyclohexane over Nanotube-Supported Pt and Pd Catalysts. Energy Fuels 2004, 18, $1429-1433$.

(41) Pande, J. V.; Shukla, A.; Biniwale, R. B. Catalytic dehydrogenation of cyclohexane over Ag-M/ACC catalysts for hydrogen supply. Int. J. Hydrogen Energy 2012, 37, 6756-6763. 